\title{
Synthese in der Geographie - Zu einer amerikanisch-deutschen Neuerscheinung
}

Peter Hagett: Geographie - Eine moderne Synthese. 767 S., zahlr. Abb., Harper \& Row/Brockhaus, stuttgart, 1983.

Ende 1983 erschien bei Harper und Row in New York die dritte Auflage von Peter Haggets "Geographie" in deutscher, von R. Hartmann, U.Meyer-Neumann, M. Preyssinger und $\mathrm{H}$. Stöckl besorger Uebersetzung mit einer Einleitung von $\mathrm{R}$. Geipel. Das Buch gilt übereinstimmend bei der englisch-sprachigen Kritik als die beste Einführung in die bestehende Gesamtdisziplin. Dies erklärt wohl auch die Uebertragung ins Deutsche, die zugleich eine "Transposition an die mitteleuropäische Situation, also für deutsche, österreichische und Schweizer Leser" sein möchte.

Das Buch wird im Untertitel als "eine moderne Synthese" bezeichnet, die "das ganze Spektrum der Geographie... in einem neuzeitlichen Zusammenhang darzustellen" versucht. Es erstrebt sie "auf zwei Ebenen: einmal, indem es die verschiedenen Traditionen und Themenbereiche der Geographie innerhalb des Gebietes verknüpft und zum zweiten, indem es die zusammensetzende Funktion der gesamten Geographie in Beziehung zu ihren Nachbarwissenschaften herausstellt." Ferner will es "den Studenten ohne vorherige geographische Ausbildung in ein Gebiet einfüren, dessen Horizonte sich mit grosser Geschwindigkeit erweitern, und das als akademisches Fach und angewandte Wissenschaft zunehmende Bedeutung erhält". Mit diesen Sätzen des Vorworts wird deutlich, worin Aufgaben und Ziele des Buches gesehen werden. Doch mag eine Durchsicht die Absichten des Verfassers noch etwas detaillierter beleuchten.

Bereits der "Prolog" bestätigt das weitgehende Gelingen des Unternehmens.

Haggett setzt in origineller Weise am "Strand" an, der als Lebensraum und Erholungsgebiet symbolische besitzt und skizziert zugleich einige"Grundbegriffe". Er macht mit den Termini "Raum", "Standort" und "Ort" bekannt, die er mit Recht als Leitworte der Geographie betrachtet.
Mit ihrer Erläuterung deren Ziele provisorisch umreissend entwirft dieser Buchabschnitt zugleich die Leitlinien erdkundlicher Forschung, wobei nach Ausführungen über die "Tyrannei von Raum und Zeit" vor allem die Beziehungen Mensch-Umwelt und wesentliche Werkzeuge der Geographie (Karte, Modell, Paradigma) Erörterung finden. Damit weitet sich der Horizont auf die "Welt jenseits des Strandes", die ganze Erdhülle.

In Teil 1 des Buches wird zunächst unter dem Titel "die Herausforderung durch die Umwelt" diese selbst vorgestellt. Es kommen die Struktur der Erdhülle (Litho-Atmo-Hydro- und Phytosphäre als Oekosysteme) und ihre Nutzungseignung (Produktionskapazität, Ressourcen, Belastbarkeit, Risiken) sowie anschliessend der Mensch nach seinen demographischen Verhältnissen zur Sprache, wobei besonders deren Dynamik (Wachstumsmuster) gedacht wird. Weiter folgt, in den Teilen 2 und 3 des Buches, mit den Kapiteln "Belastungen der Umwelt", "Erhaltung der Ressourcen" und "Veränderung des Landschaftsbildes" sowie "Entstehung regionaler Mosaiken" die Darstellung der menschlichen "Reaktionen" auf die Umwelt"; sie erfolgt mit modernsten Methoden (Hägerstrands Modelle), wobei auch die Mathematik zu ihrem Rechte kommt.

Hierauf wendet sich Haggett mit Teil 4, den "regionalen Rangordnungen" $z u$, als welche er die "Verstädterung", "stadtketten", sowie die "Welten innerhalb und ausserhalb der stadt" (Agrar-, Industrie- und Verkehrsgeographie" in knapper jedoch anregenden weise behandelt. Schliesslich folgt unter dem Titel "Internationale Spannungen und Belastungen" mit den Kapiteln "Territorium und Konflikt", "Zonenzusammenschluss und Abgrenzung", "Ungleichheiten zwischen den Staaten" und "Ungleichheiten innerhalb eines staates" eine politi-

Ernst Winkler, Prof., Dr., Mommsenstr. 2, 8044 Zürich 
sche Geographie als gleichsam krönender und die Sozialgeographie einbeziehender Abschluss der einzelen Sachgebiete der Geographie.

Die Abrundung des Werkes bildet ein "Epilog", der neue Möglichkeiten der erdkundlichen Forschung (Fernerkundung, Umweltüberwachung, Raumkartierung, Prognostik, Szenarien) und schliesslich, auf Grund einer historischen Rückschau, deren Gegenwarts- und Zukunftsproblematik erörtert. Indem zusammenfassend betont wird, dass das Buch im wesentlichen die drei Forschungsansätze der Raumanalyse, der ökologischen Systemanalyse und der Regionalkomplexanalyse vorgestellt habe, scheint es in eine ausgesprochene "Paradoxie" zum Werkuntertitel "Moderne Synthese" zu münden (zumal "Synthese nicht ausdrücklich definiert ist) und diesen Eindruck hinterlässt auch eine erste Durchsicht des Werkes. Tatsächlich herrscht in ihm jedoch ein - in mindestdoppelter Hinsicht - durchgehend synthetischer "Geist", der in der steten Verknüpfung physio- und anthropogeographischer phänomene sowie genereller wie spezieller (regionaler) Betrachtungsweise Ausdruck findet. So ist denn das Gesamtwerk eine zwar unvollständige aber im besten Sinne exemplarische Synthese, die begreiflicherweise durch ihre Beispielwahl die Herkunft aus dem angelsächsischen Sprachbereich verrät. Es darf daher als gleichermassen hervorragende wir originelle Einführung nicht nur für Studenten, sondern für alle an der Geographie Interessierten gelten; dazu tragen die reiche Illustration und die klare Sprache (an welcher die Uebersetzer offensichtlich wesentlich Anteil haben) mit den instruktiven Zusammenfassungen und Fragengruppen entschieden bei. Damit wird die eingangs erwähnte Qualifikation der Haggettschen Geographie ohne Einschränkung bekräftigt.

Im Anschluss an diese begreiflicherweise nur sehr kursorische Würdigung mögen noch einige Bemerkungen zur erdkundlichen Wissenschaftslehre erlaubt sein, die sich im Rückblick aufdrängen.

Einmal macht Haggetts Buch überzeugend klar, dass die Geographie (wie übrigens jede Wissenschaft) stets gleicherweise analytisch und synthetisch zu arbeiten hat, schärfer ausgedrückt, dass die eine ohne die andere Verfahrensweise (gar) nicht möglich ist. Das bestätigt auch die ebenso triviale wie wahre Ueberlegung, dass jede Analyse zerlegbare, jede Synthese zusammenfügbare objekte voraussetzt. Analoges gilt für die vielfache Kontroversen bereitenden sogenannten Dualismen von allgemeiner und spezieller (regionaler), nomotheti- scher und idiographischer, induktiver und deduktiver, pluralistischer und monistischer, quantitativer (mathematischer) und qualitativer Betrachtungsweise usw. Ebenso ist die verschiedenerseits geforderte Trennung der Geographie in eine naturwissenschaftliche und eine sozialwissenschaftliche (geisteswissenschaftliche) Disziplingruppe bzw. ihre Zuweisung $z u$ den Natur- und Geisteswissenschaften (welche Gegenüberstellung längst als fragwürdig erkannt wurde) ein Relativitätsproblem. Mit solchen Fragekomplexen hängen im Grunde auch das oft beklagte "Theoriedefizit" und das "Krisenbewusstsein" in der Geographie zusammen. Dass beide bestehen, wird niemand bestreiten. Sie sind indessen Charakteristika jeder Wissenschaft. Beiden liegt die Tatsache zu Grunde, dass das wissenschaftliche Erkennen "nie die wirklichkeit erschöpft" (A. Hettner), weil "wir nie Gewissheiten aussprechen können", uns "immer nur Wahrscheinlichkeiten zugänglich sind" (F. Ratzel), da alles Erkennen begrenzt ist, jede Problemlösung "neuen", weiteren Fragen ruft. Darum sollten Defizite und Krisen weniger negativ, vielmehr - wie auch das Haggettsche Werk schönstens bewahrheitet - als Dauerimpulse der steten Erneuerung oder Weiterentwicklung der Wissenschaft gewertet werden, die durch Kontroversen meist Belebung erfahren. Dieser Maxime gab schon ein "unbefangener Semantiker", der Dichter und Forscher J.W. Goethe massgebenden und wegweisenden Ausdruck: Das Grösste wäre, zu begreifen, dass alles Faktische schon Theorie ist ... Man suche nur nichts hinter den Phänomenen, sie selbst sind die Lehre.

Wir möchten bei dieser Gelegenheit festhalten, dass wir Kurzartikel und Buchbesprechungen auf Wunsch der Autoren unredigiert publizieren.

Die Redaktion 